\title{
Resecting critical nodes from an epileptogenic circuit in refractory focal-onset epilepsy patients using subtraction ictal SPECT coregistered to MRI
}

\author{
Abhijay Jalota, MD, ${ }^{1}$ Marvin A. Rossi, MD, PhD, ${ }^{1,2}$ Volodymyr Pylypyuk, CNMT, NMTCB, ARRT(N), ${ }^{2}$ \\ Michael Stein, MD, ${ }^{1}$ Travis Stoub, PhD, ${ }^{1}$ Antoaneta Balabanov, MD, ${ }^{1}$ Donna Bergen, MD, ${ }^{1}$ \\ Adriana Bermeo, MD, ${ }^{1}$ Esmeralda Park, MD, ${ }^{1}$ Michael Smith, MD, ${ }^{1}$ and Richard Byrne, MD ${ }^{3}$ \\ ${ }^{1}$ Rush Epilepsy Center, Department of Neurological Sciences; '2Department of Diagnostic Radiology and Nuclear Medicine; and \\ ${ }^{3}$ Department of Neurosurgery, Rush University Medical Center, Chicago, Illinois
}

\begin{abstract}
OBJECTIVE The purpose of this study was to assess the positive predictive value of postresection outcomes obtained by presurgical subtracted ictal SPECT in patients with lesional (MRI positive) and nonlesional (MRI negative) refractory extratemporal lobe epilepsy (ETLE) and temporal lobe epilepsy (TLE). Specifically, outcomes were compared between partial versus complete resection of the regions of transient hyperperfusion identified using subtraction ictal SPECT coregistered to MRI (SISCOM) in relation to the ictal onset zone (IOZ) that was confirmed by electrocorticography $(E C O G)$. That is, SISCOM was used to understand the long-term postsurgical outcomes following resection of the IOZ that overlapped with 1 or more regions of ictal onset-associated transient hyperperfusion.

METHODS The study cohort included 44 consecutive patients with refractory ETLE or TLE who were treated between 2002 and 2013 and underwent presurgical evaluation using SISCOM. Concordance was determined between SISCOM localization and the IOZ on the basis of ECoG monitoring. In addition, the association between the extent of the resection site overlapping with the SISCOM signal and postresection outcomes were assessed. Postsurgical follow-up was longer than 24 months in 39 of 44 patients.
\end{abstract}

RESULTS The dominant SISCOM signals were concordant with ECoG and overlapped the resection site in 32 of 44 (73\%) patients (19 ETLE and 13 TLE patients), and 20 of $32(63 \%)$ patients became seizure free. In all 19 ETLE patients with concordant SISCOM and ECoG results, the indicated location of ictal onset on ECoG was completely resected; 11 of 19 patients (58\%) became seizure free (Engel Class I). In all 13 TLE patients with concordant SISCOM and ECoG results, the indicated ECoG focus was completely resected; 9 of 13 patients (69\%) became seizure free (Engel Class I). Complete resection of the SISCOM signal was found in 7 of 34 patients (21\%). Of these 7 patients, 5 patients $(72 \%)$ were seizure free (Engel Class I). Partial resection of the SISCOM signal was found in 16 of 34 patients (47\%), and 10 of these 16 patients (63\%) were seizure free (Engel Class I) after more than 24 months of follow-up.

CONCLUSIONS Concordance between 1 or more SISCOM regions of hyperperfusion with ECoG and at least partial resection of the dominant SISCOM signal in this refractory epilepsy cohort provided additional useful information for predicting long-term postresection outcomes. Such regions are likely critical nodes in more extensive, active, epileptogenic circuits. In addition, SPECT scanner technology may limit the sensitivity of meaningful SISCOM signals for identifying the maximal extent of the localizable epileptogenic network.

http://thejns.org/doi/abs/10.3171/2015.6.JNS141719

KEY WORDS ictal onset zone; electrocorticography; temporal lobe epilepsy; subtraction ictal SPECT coregistered to MRI; epileptogenic network; extratemporal epilepsy; magnetic resonance imaging

$\mathrm{E}$ PILEPSY is estimated to affect between 2.5 and 3 million people in the United States, with a prevalence of 5 to 10 persons per $1000 .{ }^{32}$ It has been shown that about $47 \%$ of patients with a new diagnosis of focal-onset epilepsy are successfully treated with the first antiepileptic drug (AED) prescribed. ${ }^{19}$ Therapeutic failure of 2 AEDs is defined as drug-resistant epilepsy. ${ }^{2,5}$ Approximately $20 \%$ to $40 \%$ of patients with epilepsy are likely to have drug-resistant epilepsy. ${ }^{4,26,27}$ Patients with a unilateral lesion on high-resolution MRI that is concordant with the

ABBREVIATIONS aECOG = acute electrocorticography; $A E D=$ antiepileptic drug; $E C O G$ = chronic electrocorticography; EEG = electroencephalography; ETLE = extratemporal epilepsy; IOZ = ictal onset zone; SISCOM = subtraction ictal SPECT coregistered to MRI; SPGR = spoiled gradient recalled; TLE = temporal lobe epilepsy. SUBMITTED July 28, 2014. ACCEPTED June 17, 2015.

INCLUDE WHEN CITING Published online March 18, 2016; DOI: 10.3171/2015.6.JNS141719. 
electroencephalography (EEG) findings and seizure semiology have the best outcomes after resection. . $^{13,31,34} \mathrm{Un}$ fortunately, 20\% to 30\% of temporal lobe epilepsy (TLE) patients and $20 \%$ to $50 \%$ of extratemporal lobe epilepsy (ETLE) patients demonstrate the absence of an underlying lesion on high-resolution MRI. ${ }^{6,16,18}$ Resective targeting of the ictal onset zone (IOZ) is most challenging in patients with nonlesional MRI and focal-onset epilepsy. Previous postresection outcome studies report seizure freedom to be the greatest after resection in lesional TLE patients, where $75 \%$ to $85 \%$ of patients achieve Engel Class I after 2 years of follow-up (Engel classification: Class I, seizure free; Class II, rare disabling seizures; Class III, worthwhile improvement; and Class IV no worthwhile improvement). ${ }^{3,9}$ Patients with lesional ETLE have seizure-free rates (Engel Class I) of around $60 \%$ (at 1-year follow-up) ${ }^{31}$ In contrast, patients with nonlesional TLE demonstrate outcomes with seizure-free rates (Engel Class I) ranging from $41 \%$ to $65 \%$ (at 2-year follow-up). ${ }^{1,14,24,30}$ Patients with nonlesional ETLE exhibit the lowest seizure-free outcomes, ranging from $24 \%$ to $42 \%$ (at 2-year follow-up) ${ }^{8,20,23} \mathrm{~A}$ better understanding is necessary to evolve our approach toward recognizing the IOZ as a critical node in a larger epileptogenic network in order to achieve better seizure-free rates, particularly in nonlesional focal-onset epilepsy.

In the present study, we retrospectively analyzed the clinical history profiles including duration of epilepsy, video EEG (noninvasive and invasive) and MRI findings, subtraction ictal SPECT coregistered to MRI (SISCOM) data, seizure semiology, sites and sizes of resection, pathological findings, and postsurgical seizure outcomes in a cohort of patients with lesional and nonlesional ETLE and TLE. The aim of our study was to assess the positive predictive value of resecting SISCOM regions of transient hyperperfusion that were associated with the IOZ. Also, the long-term postsurgical outcomes in patients with both MRI-positive and MRI-negative ETLE and TLE were compared.

\section{Methods \\ Patients}

A total of 567 presurgical SPECT studies (243 patients) were performed at our center between 2002 and 2013. Our study included a cohort of 44 patients from this presurgical group who underwent resective epilepsy surgery. The mean age of the patients in our cohort was 29 years (range 11-57 years; SEM 1.8) (Table 1). Patients underwent follow-up for a mean of 5 years (range 2-10 years). All patients in our cohort were assessed using chronic intracranial electrode electrocorticography (ECoG) monitoring and/or acute intraoperative electrocorticography $(\mathrm{aECoG})$. The placement of the electrodes for invasive monitoring was determined during an epilepsy surgery management conference at our institution. Multiple factors were analyzed to generate the hypothesis for intracranial electrode placement and targeting the IOZ. To facilitate localization of the IOZ, we employed seizure semiology, MRI, chronic noninvasive scalp video-EEG monitoring, magnetoencephalography, SISCOM, positron emission tomography, and neurocognitive testing (Table 2).
TABLE 1. Characteristics of the study participants

\begin{tabular}{lc}
\hline \multicolumn{1}{c}{ Characteristic } & Value* $^{*}$ \\
\hline Resections & 44 \\
\hline Age (yrs) & $11-57$ \\
\hline Range & 29 \\
\hline Mean & $23(52 \%)$ \\
\hline Male & $21(48 \%)$ \\
\hline Female & $38(86 \%)$ \\
\hline Rt-handed & $17(38 \%)$ \\
\hline Lesional temporal epilepsy & $1(2 \%)$ \\
\hline Nonlesional temporal epilepsy & $14(32 \%)$ \\
\hline Lesional extratemporal epilepsy & $12(27 \%)$ \\
\hline Nonlesional extratemporal epilepsy
\end{tabular}

* Values are shown as $n(\%)$ unless otherwise stated.

We included patients based on the following inclusion criteria: 1) diagnosis of drug-resistant, focal-onset epilepsy based on presurgical evaluation that included SISCOM; and 2) resective epilepsy surgery performed with a postoperative follow-up of $\geq 24$ months (mean followup 5 years). The predominant reasons for exclusion from the study were 1) patient underwent less than 24 months of follow-up (except patients who remained as Engel Class IV [no worthwhile improvement]), or 2) no interictal SPECT was acquired. Forty-four patients met all of the inclusion criteria and were analyzed in this study. One ETLE patient in the study had undergone a previous resection prior to a second resection. In 25 (61\%) patients, postresection MRI was performed. Preresection subtraction ictal SPECT was coregistered to the patient's postresection MRI study, which was acquired at least 12 months postoperatively. This technique accounted for brain shift following resection. This strategy was used to determine the regions of transient hyperperfusion in comparison with the extent of resection.

\section{High-Resolution MRI}

High-resolution, gapless, pre- and postgadolinium, volumetric, spoiled gradient recall (SPGR) MR images of the brain were obtained for each patient. The MRI examination was performed using a 1.5- or 3-T scanner (Siemens). A gapless, SPGR, T1-weighted sequence data set for each patient was acquired at a slice thickness of 1.0 or $1.6 \mathrm{~mm}$.

\section{Noninvasive Video-EEG Monitoring}

Chronic, noninvasive video-EEG monitoring was performed in each patient using the International 10-20 system for electrode placement, including anterior temporal and sphenoidal electrodes if appropriate. AEDs were lowered during chronic monitoring, all patients were electrographically monitored to record the typical seizures, and video recordings were generated to monitor semiology.

\section{SISCOM}

SPECT was performed on each patient during videoEEG monitoring using Ceretec ( $\left.{ }^{99 \mathrm{~m}} \mathrm{Tc}-\mathrm{HMPAO}\right)$ or Neurolite $\left({ }^{99 \mathrm{~m} T c-E C D)}\right.$ ) at a dose of 24 to $26 \mathrm{mCi}$ (adjusted for 


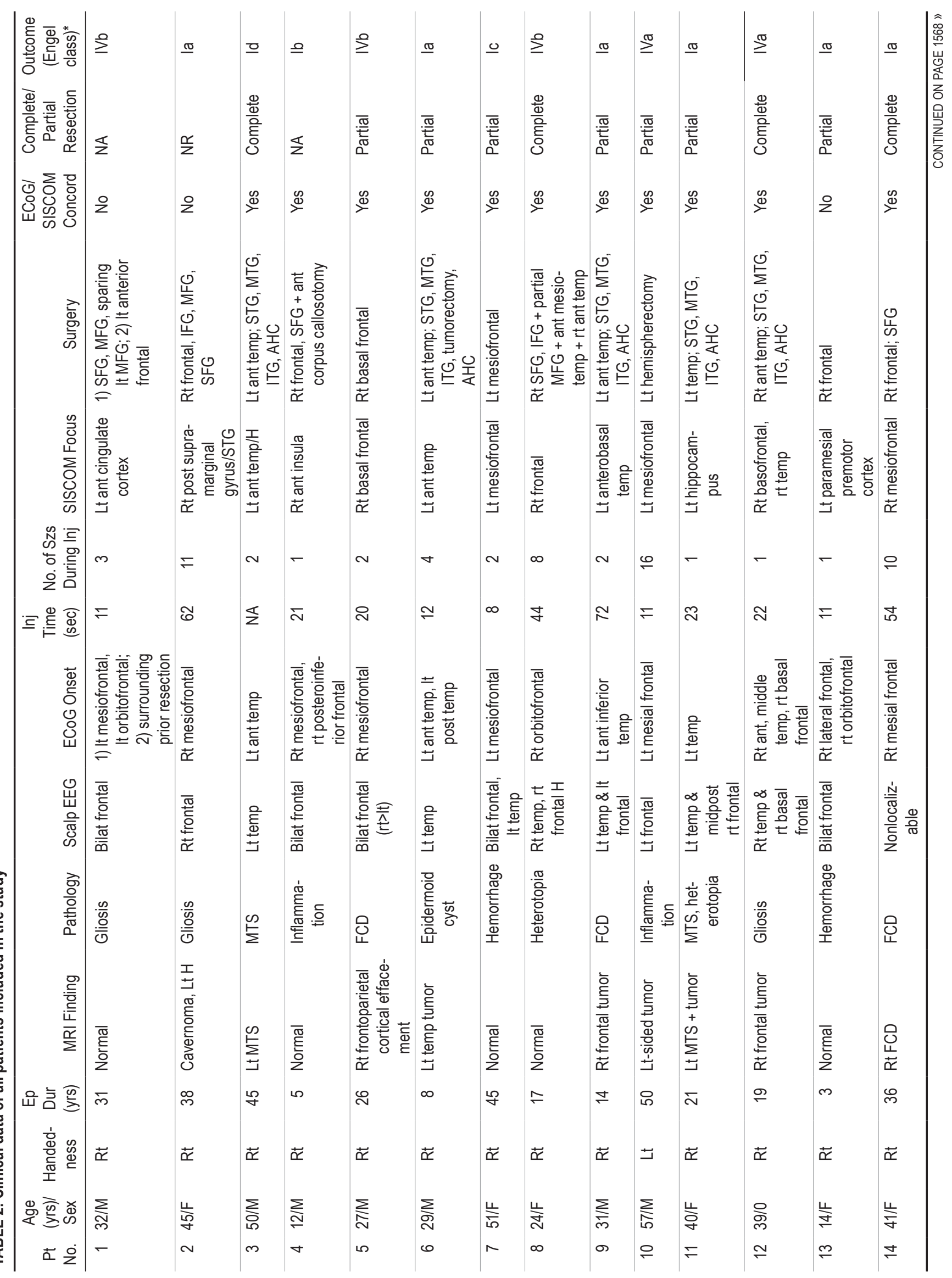




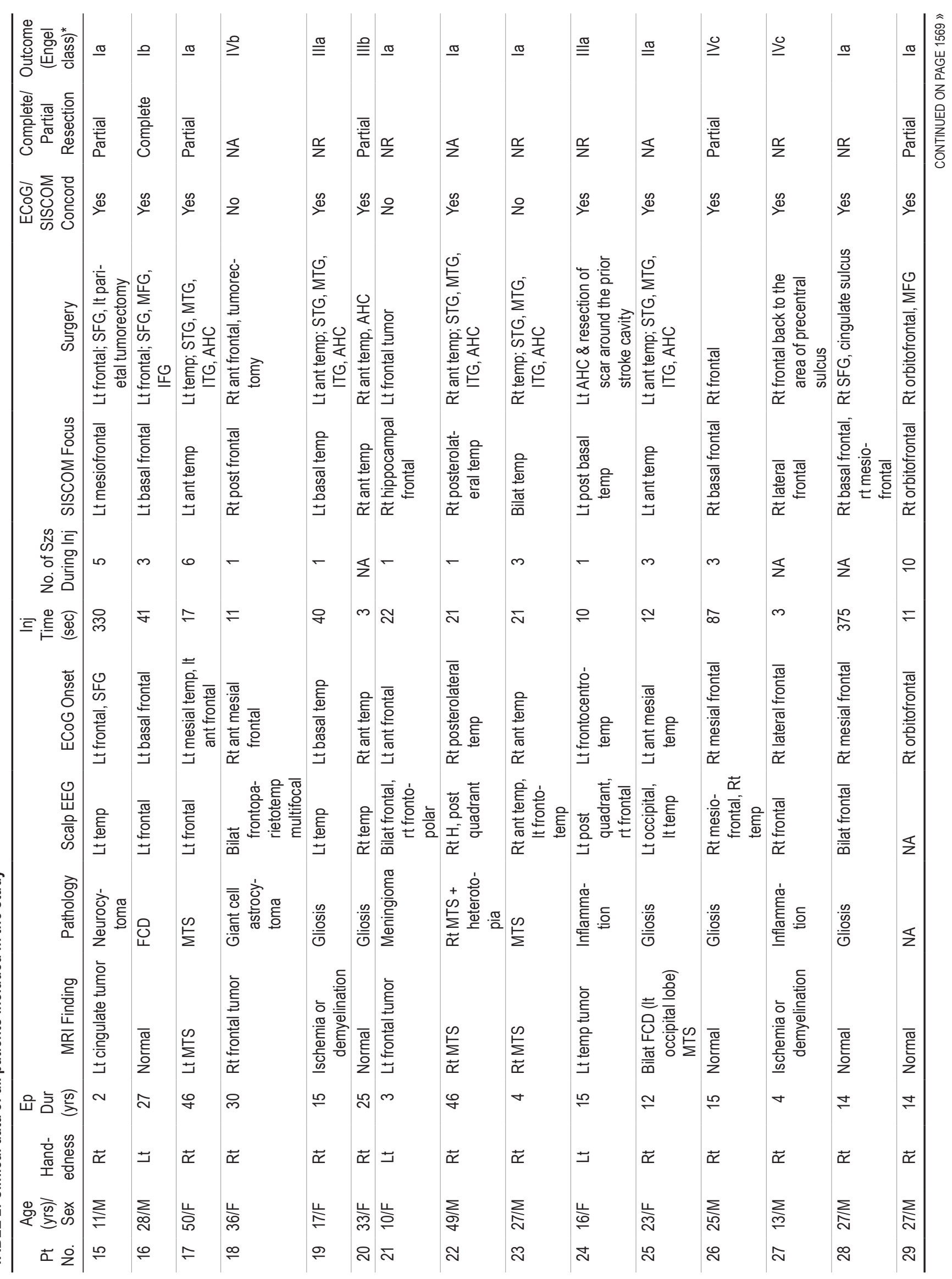




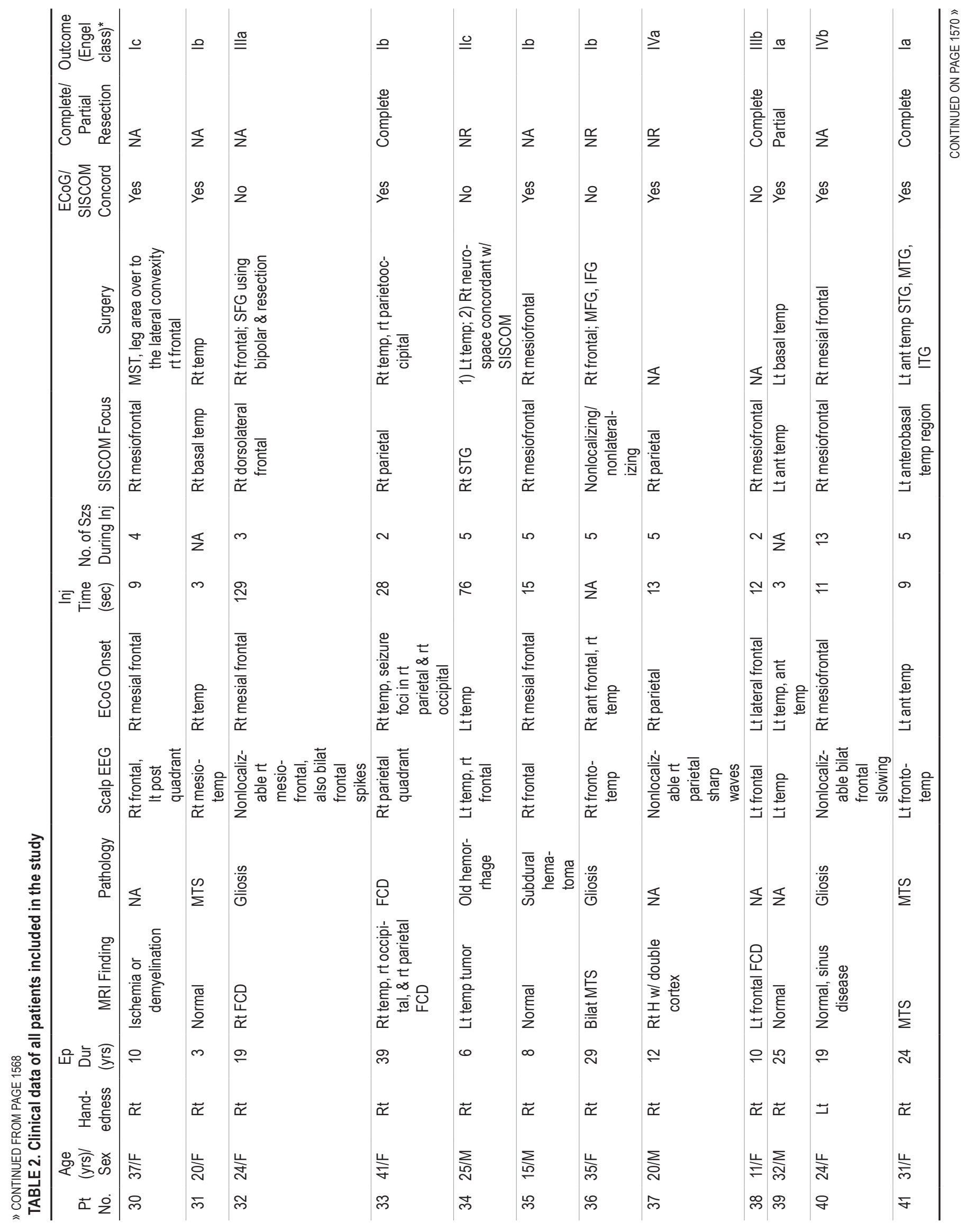




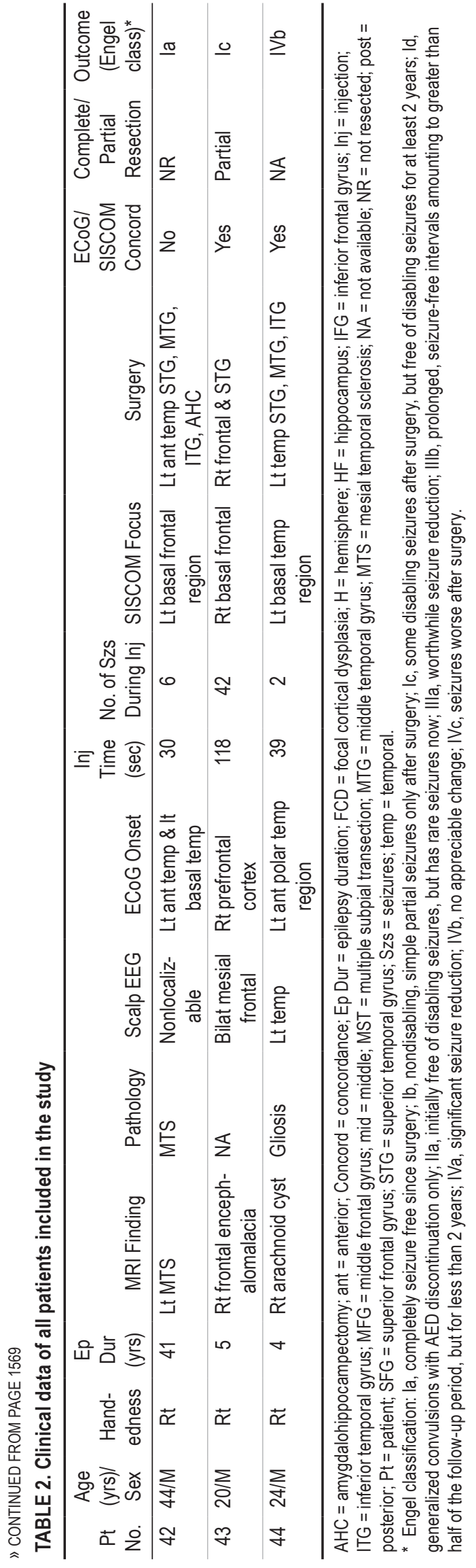

body weight). The radiotracer was injected for the ictal SPECT studies with an autoinjector (MedRad) used by a specially trained nuclear medicine technologist (V.P.) while at the patient's bedside during seizure onset. The technologist was trained to inject at the clinical and/or electroencephalographic seizure onset, whichever came first. The seizure-onset and ending times were always noted. Also, the time from the beginning of injection up to the complete depression of the syringe plunger was noted. We retrospectively calculated the time of the radiotracer injection following seizure onset by reviewing the video-EEG recordings for all patients. To avoid injection during the postictal state, a minimum of 15 seconds was required between the end of radiotracer injection and the end of the seizures, as described above. Baseline SPECT was obtained by performing an interictal injection after a seizure-free period of $\geq 24$ hours. Brain SPECT scans were obtained within 2 hours after injection using a Siemens E.CAM Dual-Detector gamma camera with BiOCORE fan beam collimators. Thirty-two views per detector (total 64) were acquired using 80,000 counts per view, circular orbit, and "step and shoot" mode. Images were reconstructed by Syngo Siemens AG software using a $128 \times 128$ matrix with a Butterworth filter. DICOM isotropic datasets with a voxel size of 3.9 $\mathrm{mm}$ were created and transferred to Analyze software (AnalyzeDirect). Analyze version 10 was used to perform ictal and interictal SPECT thresholding, normalization, subtraction, and coregistration to the patient's MRI study. Finally, the brain was segmented from the extracerebral structures, as identified on the patient's volumetric MRI study. The cerebral surface of the binary ictal SPECT was matched to the cerebral surface of the binary MRI. The resulting registered transformation matrix was then applied to the subtraction SPECT data set in order to coregister it to the processed MRI study. Complete, gapless, SISCOM image sets were presented to a blinded, board-certified epilepsy neurologist (M.A.R.) who determined the SISCOM region of transient hyperperfusion for each patient in this study.

\section{Invasive Recording}

The ECoG electrode placement plan (subdural grid electrodes and/or depth electrodes) was confirmed using head CT. In 41 patients, chronic bedside ECoG monitoring was performed to investigate the clinical electrocerebral IOZ based on the patient's stereotypic seizure. For 3 patients, aECoG monitoring alone provided enough information to establish the IOZ.

\section{Pathology}

A pathological diagnosis of the resected specimens, such as gliosis, focal cortical dysplasia, heterotopias, tumor, or mesial temporal sclerosis, was determined for 38 of $44(86 \%)$ patients (Table 2).

\section{Postoperative Seizure Outcomes}

Postoperative outcomes were determined by following 38 patients for a period greater than 24 months (mean follow-up 5 years; range 2-10 years) after resection. We classified the postresection outcomes into 4 classes and subclasses according to Engel's classification. ${ }^{11}$ At the time 
of our analysis, 5 patients in our study received a followup period of 17 to 18 months. However, these patients remained classified as Engel Class IV. One of the patients in our study was observed for only 12 months. However, this patient remained in Engel Class IVc (no worthwhile improvement, worsening of seizures after surgery).

\section{Postresection MRI}

We obtained postresection MRIs for 34 of $44(77 \%)$ patients. We coregistered the preresection SISCOM on postresection MRI in order to evaluate the extent of resection of the identified SISCOM region of hyperperfusion. Using traditional SISCOM processing with Analyze, we could not achieve accurate registration of the preresection SISCOM signals on the postresection MRI studies for all patients. Postresection MRI findings were considerably different from preresection MRI findings due to the creation of new cortical boundaries, particularly in the area of resection. In addition, the craniotomy flap altered the skull shape in some patients. Consequently, clean object extraction could not be achieved in several postresection MRI data sets, resulting in poor registration. To solve this problem, we employed a different postprocessing method. We performed 3-dimensional voxel registration using Analyze between the original fused preresection SISCOM, which was used as the base, and the postresection MRI, which was used as the match. We first performed manual registration to achieve similar alignment in all 3 planes (transverse, coronal, and sagittal). We then performed autoregistration using the register tab in the 3-dimensional voxel registration window in Analyze. This process perfectly aligned the two 3-dimensional objects, so that each voxel in the new fused image represented the same voxel of the base (preresection SISCOM) and match (postresection MRI).

The intensities of the base and match were modified as follows. The minimum intensity of the base image was increased in order to make the preresection MRI disappear and reduce the maximum of the base in order to improve the SPECT signal. This technique prevented the preresection brain tissue boundary from filling the resection site. Thus, the fused image demonstrated the properties of the SPECT signal of the base, but not the MRI properties of the base. Lastly, we reduced the maximum intensity of the match to intensify the postresection MRI. This technique enabled us to better visualize the resection site. This process resulted in the accurate registration of the preresection, SISCOM hyperperfusion signals over the postresection MRI, and, at the same time, improved the intensity of the resection site.

\section{Analysis and Statistics}

Concordance was determined between SISCOM localization and ECoG on the basis of an overlapping IOZ and called "concordance with the ECoG." Concordance was also determined between SISCOM localization and the resection site, or "concordance with the resection site." Two different concordance types are designated since resection usually involves a larger area than the IOZ, as determined by ECoG. Strict concordance criteria were used, where $>90 \%$ of the resected area must overlap with the SISCOM signal to be considered concordant. Concor- dance was determined by a blinded, board-certified epilepsy neurologist (M.A.R.).

We coregistered preresection and subtracted ictal SPECT on postresection MRI to determine if the SISCOM signal was completely or partially removed. We determined if complete or partial resection impacted postoperative outcomes.

Our cohort of 44 patients underwent resective epilepsy surgery. All patients underwent follow-up for at least 2 years (mean follow-up 5.0 years; range 2-10 years). Five patients only received 17 to 18 months of follow-up at the time of our analysis, but these patients demonstrated Engel Class IV outcomes. One of the patients completed a follow-up of only 12 months, but achieved Engel Class IVc. The mean radiotracer injection time for all patients was 45 seconds (SEM 11.4) after electrocerebral seizure onset. In our cohort, all SPECT studies were performed during the patient's stereotypic seizures, as measured electrographically or clinically.

\section{Results \\ ECoG Monitoring}

ECoG concordant tissue was completely resected from all 44 patients. Of these 44 patients, 24 of $44(55 \%)$ became seizure free (Engel Class I) after at least 2 years of follow-up after resection.

\section{SISCOM Concordance}

SISCOM was concordant with ECoG and the resection site in 32 of $44(73 \%)$ patients, and 20 of these $32(63 \%)$ became seizure free (Engel Class I after $>2$ years followup). Of these 32 patients, 19 of $32(59 \%)$ were ETLE and 13 of 32 (41\%) were TLE (Fig. 1). The mean injection time was 46 seconds in the SISCOM ECoG concordant group (SEM 14.5).

\section{SISCOM Concordant ETLE Group}

In 19 ETLE patients with concordant SISCOM and ECoG results, ECoG delineated the epileptogenic source or focus, which was completely resected in all patients; 11 of 19 patients $(58 \%)$ became seizure free (Engel Class I) (Table 3).

Among these 19 ETLE patients, 8 patients exhibited a lesion on MRI (focal cortical dysplasia, tumor, double cortex, or encephalomalacia). Only 6 of these 8 patients exhibited an MRI lesion and SISCOM focus that were both concordant with the ECoG results; only 3 of these 6 patients $(50 \%)$ became seizure free (Engel Class I) (Table 3).

Also, among these 19 ETLE patients, 11 patients were MRI negative for lesions. For these patients, SISCOM facilitated the placement of the intracranial electrodes; 7 (64\%) patients became seizure free (Engel Class I) (Table 3).

\section{SISCOM Concordant TLE Group}

In the 13 TLE patients with concordant SISCOM and ECoG results, the indicated ECoG focus was completely resected; 9 of 13 patients $(69 \%)$ became seizure free (Engel Class I) (Table 3).

Among these 13 TLE patients, 11 patients had a lesion 
TABLE 3. SISCOM concordant with ECOG and overlapping with the resection site in ETLE $(n=19)$ and TLE $(n=13)^{*}$

\begin{tabular}{|c|c|c|c|c|c|c|}
\hline \multirow[b]{2}{*}{$\begin{array}{l}\text { Engel } \\
\text { Class }\end{array}$} & \multicolumn{3}{|c|}{ ETLE } & \multicolumn{3}{|c|}{ TLE } \\
\hline & $\begin{array}{l}\text { SISCOM \& Lesional } \\
\text { MRI Concordant } \\
\text { w/ aECoG/ECoG } \\
(n=6)\end{array}$ & $\begin{array}{l}\text { SISCOM Concordant, } \\
\text { Lesional MRI } \\
\text { Nonconcordant w/ } \\
\text { aECoG/ECoG }(n=2)\end{array}$ & $\begin{array}{c}\text { SISCOM Concordant } \\
\text { w/ aECoG/ECoG, } \\
\text { Nonlesional MRI } \\
(n=11)\end{array}$ & $\begin{array}{l}\text { SISCOM \& Lesional } \\
\text { MRI Concordant } \\
\text { w/ aECoG/ECoG } \\
(n=8)\end{array}$ & $\begin{array}{l}\text { SISCOM Concordant, } \\
\text { Lesional MRI } \\
\text { Nonconcordant w/ } \\
\text { aECoG/ECoG }(n=3)\end{array}$ & $\begin{array}{l}\text { SISCOM Concordant } \\
\text { w/ aECoG/ECoG, } \\
\text { Nonlesional MRI } \\
(n=2)\end{array}$ \\
\hline I & $3(50 \%)$ & $1(50 \%)$ & 7 (64\%) & $7(88 \%)$ & $1(33 \%)$ & $1(50 \%)$ \\
\hline II & 0 & 0 & 0 & 0 & $1(33 \%)$ & 0 \\
\hline III \& IV & $3(50 \%)$ & $1(50 \%)$ & $4(36 \%)$ & $1(12 \%)$ & $1(33 \%)$ & $1(50 \%)$ \\
\hline
\end{tabular}

${ }^{*}$ Values are shown as $n(\%)$.

(mesial temporal sclerosis, focal cortical dysplasia, or tumor) on MRI. Only 8 of these 11 patients demonstrated an MRI lesion and SISCOM focus that were both concordant with the ECoG results; 7 of these 8 patients $(88 \%)$ became seizure free (Engel Class I) (Table 3).

Also, among these 13 TLE patients, 2 patients were MRI negative. For these patients, SISCOM guided the placement of the intracranial electrodes; $1(50 \%)$ patient became seizure free (Engel Class I) (Table 3).

\section{Nonconcordant Group}

SISCOM was nonconcordant with ECoG but overlapped with the resection site in only 1 of 44 patients. This patient was diagnosed with nonlesional ETLE but did not exhibit a lesion on MRI. This patient demonstrated a worse outcome after resection and achieved Engel Class IV ( $>2$ years of follow-up, Fig. 1).

SISCOM was nonconcordant with ECoG and nonoverlapping with the resection site in 11 of 44 patients (Fig.

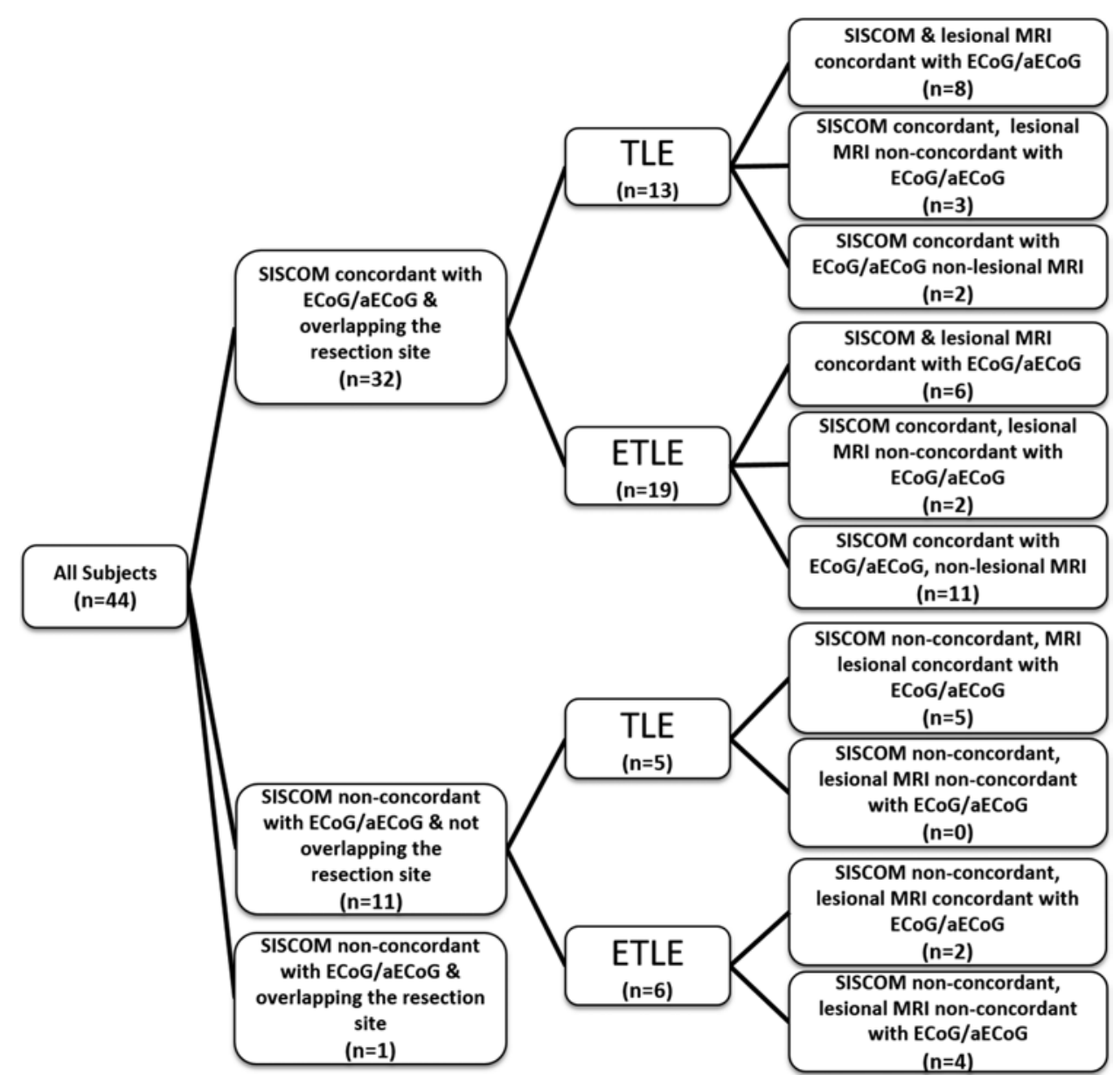

FIG. 1. Classification hierarchy of patient outcomes after resective epilepsy surgery. Three main groups are represented: 1) SISCOM concordant with ECOG and overlapping with the resection site; 2) SISCOM nonconcordant with ECOG and nonoverlapping with the resection site in TLE patients; and 3) SISCOM nonconcordant with ECOG and overlapping with the resection site. 
TABLE 4. Outcomes for SISCOM nonconcordant with aECoG/ECoG and nonoverlapping with the resection site $(n=11)^{*}$

\begin{tabular}{|c|c|c|c|c|}
\hline \multirow[b]{2}{*}{ Engel Class } & \multicolumn{2}{|c|}{ ETLE } & \multicolumn{2}{|c|}{ TLE } \\
\hline & $\begin{array}{l}\text { SISCOM Nonconcordant, } \\
\text { Lesional MRI Concordant w/ } \\
\text { aECoG/ECoG }(n=2)\end{array}$ & $\begin{array}{l}\text { SISCOM Nonconcordant, MRI } \\
\text { Lesional Nonconcordant w/ } \\
\text { aECoG/ECoG }(n=4)\end{array}$ & $\begin{array}{l}\text { SISCOM Nonconcordant, } \\
\text { MRI Lesional Concordant w/ } \\
\text { aECoG/ECoG }(n=5)\end{array}$ & $\begin{array}{l}\text { SISCOM Nonconcordant, } \\
\text { Lesional MRI Nonconcordant w/ } \\
\text { aECoG/ECoG }(n=0)\end{array}$ \\
\hline I & $1(50 \%)$ & $1(25 \%)$ & $2(40 \%)$ & 0 \\
\hline II & 0 & 0 & $1(20 \%)$ & 0 \\
\hline III \& IV & $1(50 \%)$ & $3(75 \%)$ & $2(40 \%)$ & 0 \\
\hline
\end{tabular}

* Values are shown as $\mathrm{n}(\%)$.

1). In the nonconcordant group, 4 of $11(36 \%)$ patients achieved Engel Class I, 1 of $11(9 \%)$ patients achieved Engel Class II (rare disabling seizures), 2 of 11 (18\%) patients achieved Engel Class III (worthwhile improvement), and 4 of $11(36 \%)$ patients achieved Engel Class IV. All of these 11 patients exhibited lesions on MRI (Table 4).

For the SISCOM nonconcordant group, the average injection time was 37 seconds (SEM 11.3).

\section{Resection of SISCOM Focus}

We coregistered the preresection subtraction ictal SPECT to each patient's postresection MRI in order to determine if the SISCOM signal(s) used to guide the placement of the intracranial electrodes preresection was completely, partially, or not resected. A $10 \%$ shifting of the adjacent brain postresection was assumed in the postresection MRI coregistrations. Postresection MRI studies were completed in 35 of $44(80 \%)$ patients.

Complete resection of the SISCOM signal was found in 7 of 34 patients $(21 \%)$. Of these 7 patients, 5 patients $(72 \%)$ were seizure free (Engel Class I). Partial resection of the SISCOM signal was found in 16 of 34 patients (47\%); 10 of these 16 patients (63\%) were seizure free (Engel Class I) after more than 24 months of follow-up (Table 5).

The SISCOM signal was not resected in 11 of 34 (32\%) patients. Four of these 11 patients $(36 \%)$ were seizure free (Engel Class I) after more than 24 months of follow-up.

\section{Pathology}

The pathological findings (gliosis and focal cortical dysplasia) ( $\mathrm{p}=0.1007)$ were not significantly correlated with the outcomes of epilepsy surgery (Table 2).

\section{Discussion}

The success of epilepsy surgery depends on the accurate anatomical localization of the IOZ, which is indispensable to contributing to favorable outcomes. The IOZ is a com- ponent of the ictal circuitry that is critically involved in the initiation of seizure. Scalp EEG and semiology are often not sensitive enough to accurately delineate the IOZ in rapidly propagating seizures. Consequently, the development of novel neuroimaging approaches is required to facilitate visualizing the IOZ, particularly in patients with ETLE. SISCOM can help identify the IOZ, though its limitations as an indirect measure of increased metabolic demand in the nearby epileptogenic cortex must be kept in mind. The probability of seizure freedom in our cohort is highest for lesions identified on MRI. Patients with MRI findings showing lesions in 1 temporal lobe that are concordant with ipsilateral ictal activity on EEG have the highest rate of seizure freedom after resective epilepsy surgery. ${ }^{13,31}$ In our cohort, 7 patients demonstrated underlying lesions in 1 temporal lobe (predominantly mesial temporal sclerosis), and a concordant abnormality on scalp EEG was noted in 6 of these patients; all 7 of $7(100 \%)$ patients achieved Engel Class I with longer than 3 years follow-up (mean follow-up 4.9 years). SISCOM was concordant with the resection site in 6 of $8(75 \%)$ of these patients.

It has been previously shown that the probability of seizure freedom (Engel Class I) at the 12-month follow-up after surgery is 2.7 times higher in TLE patients with MRI lesions in comparison with TLE patients without MRI lesions. ${ }^{31}$ Moreover, the probability of seizure freedom (Engel Class I) at the 12-month follow-up after surgery is 2.9 times higher in ETLE patients with MRI lesions in comparison with ETLE patients without MRI lesions. ${ }^{31}$ SISCOM impacted our postresection outcomes in the nonlesional ETLE and TLE patient groups. Also, the complete or partial resection of the SISCOM region of transient hyperperfusion plays a role in seizure freedom; 5 of $7(72 \%)$ patients were seizure free after complete resection of SISCOM hyperperfusion-related signaling, and 10 of 16 $(63 \%)$ patients were seizure free after the partial resection of similar SISCOM signals. In our study, 6 patients exhibited SISCOM signal foci localized with the resection site;

TABLE 5. Outcomes in patients with partial resection of the SISCOM focus in ETLE $(n=10)$ and TLE $(n=6)^{*}$

\begin{tabular}{|c|c|c|c|c|c|c|}
\hline $\begin{array}{l}\text { Engel } \\
\text { Class }\end{array}$ & $\begin{array}{c}\text { ETLE, Lesional MRI } \\
\text { Concordant w/ aECoG/ } \\
\text { ECoG }(n=2)\end{array}$ & $\begin{array}{l}\text { ETLE, Lesional MRI } \\
\text { Nonconcordant w/ } \\
\text { ECoG }(n=2)\end{array}$ & $\begin{array}{l}\text { ETLE, MRI } \\
\text { Nonlesional (n } \\
=6 \text { ) }\end{array}$ & $\begin{array}{c}\text { TLE, Lesional MRI } \\
\text { Concordant w/ aECoG/ } \\
\text { ECoG }(n=4)\end{array}$ & $\begin{array}{l}\text { TLE, Lesional MRI } \\
\text { Nonconcordant w/ } \\
\text { aECoG/ECoG }(n=1)\end{array}$ & $\begin{array}{c}\text { TLE, MRI } \\
\text { Nonlesional }(n=1)\end{array}$ \\
\hline I & $1(50 \%)$ & $1(50 \%)$ & $4(67 \%)$ & $3(75 \%)$ & 1 & 0 \\
\hline II & 0 & 0 & 0 & 0 & 0 & 0 \\
\hline III-IV & $1(50 \%)$ & $1(50 \%)$ & $2(33 \%)$ & $1(25 \%)$ & 0 & 1 \\
\hline
\end{tabular}

* Values are shown as $n(\%)$. 
these patients demonstrated poor surgical outcomes (Engel Class IV), and at least 1 SISCOM region not overlapping with the resection site (Fig. 2). Concordance between SISCOM with ECoG had a sensitivity of $72.7 \%$, specificity of $100 \%$, positive predictive value of $100 \%$, and negative predictive value of $14.3 \%$.

Improved outcomes were seen with earlier surgery for intractable frontal lobe epilepsy. ${ }^{28}$ In our study, the mean duration of frontal lobe epilepsy was 19.17 years. When the duration of frontal lobe epilepsy was $<5$ years, $75 \%$ became seizure free $(n=4 ; 3$ MRI lesion-positive and 1 MRI lesion-negative patients) (Engel Class I, mean year of follow-up postresection 7.7 years, range 6-10 years, SD 2.08). However, when the duration of frontal lobe epilepsy was $\geq 5$ years, $59 \%$ became seizure free $(n=17 ; 7$ MRI lesion-positive and 10 MRI lesion-negative patients) (Engel Class I, mean year of follow up postresection 7.3 years, range 3-11, SD 2.8).

According to previously published success rates for partial frontal lobectomy, favorable outcomes (Engel Class I) range from $15 \%$ to $35 \%$ in these trials., ${ }^{72,20,25,29,36}$ The only variable that was significantly associated with a favorable surgical outcome was a focal MRI abnormality in frontal lobe epilepsy.$^{13}$ Elsharkawy et al. published a retrospective study investigating 97 patients with lesional frontal lobe epilepsy who underwent resection. ${ }^{10}$ This study reported the seizure freedom rates (Engel Class I) to be $54.6 \%$ at 6 months, $49.5 \%$ at 2 years, $47 \%$ at 5 years, and $41.9 \%$ at 10 years..$^{10}$ In yet another series, $41 \%$ of nonlesional TLE patients showed excellent outcomes versus $72 \%$ when an MRI abnormality was present. ${ }^{21}$ Previous studies that report favorable (Engel Class I) outcomes investigated patients with frontal lobe tumors or focal cortical dysplasias. ${ }^{36}$ Other factors that predict seizure recurrence include incomplete resection of the epileptogenic lesion, 7,10,17,33,36 predominantly generalized or poorly localized ictal EEG patterns on surface EEG, ${ }^{10,17,35}$ the lack of a subdural grid evaluation, ${ }^{10,17}$ acute postresection seizures, ${ }^{6}$ postoperative persistence of prolonged auras, ${ }^{10,17}$ history of febrile seizures, ${ }^{21}$ and the lack of a distinct MRI lesion. ${ }^{7,10,17,22,33,35}$

The SISCOM results in our study patients influenced the planning of intracranial electrode placement. Therefore, our ECoG results were, in part, influenced by our SISCOM data. Also, all patients in our cohort underwent SISCOM as part of the presurgical evaluation. Therefore, potential selection bias toward patients with more localizable IOZ has to be regarded, and consequently resulted in more favorable postresection outcomes.

\section{Conclusions}

The goal of resective epilepsy surgery is long-term seizure freedom (Engel Class I or II). Seizure outcomes are time dependent and dynamic. All prognostic factors converge into one central theme: an epileptogenic zone that is well visualized and also electrically and semiologically restricted, in that its extent offers the best chance of seizure freedom when resected completely. However, SISCOM provides an indirect measure of localizing the IOZ, which often coincides with the critical nodes within the epileptogenic network.

These data confirm previous studies that demonstrate

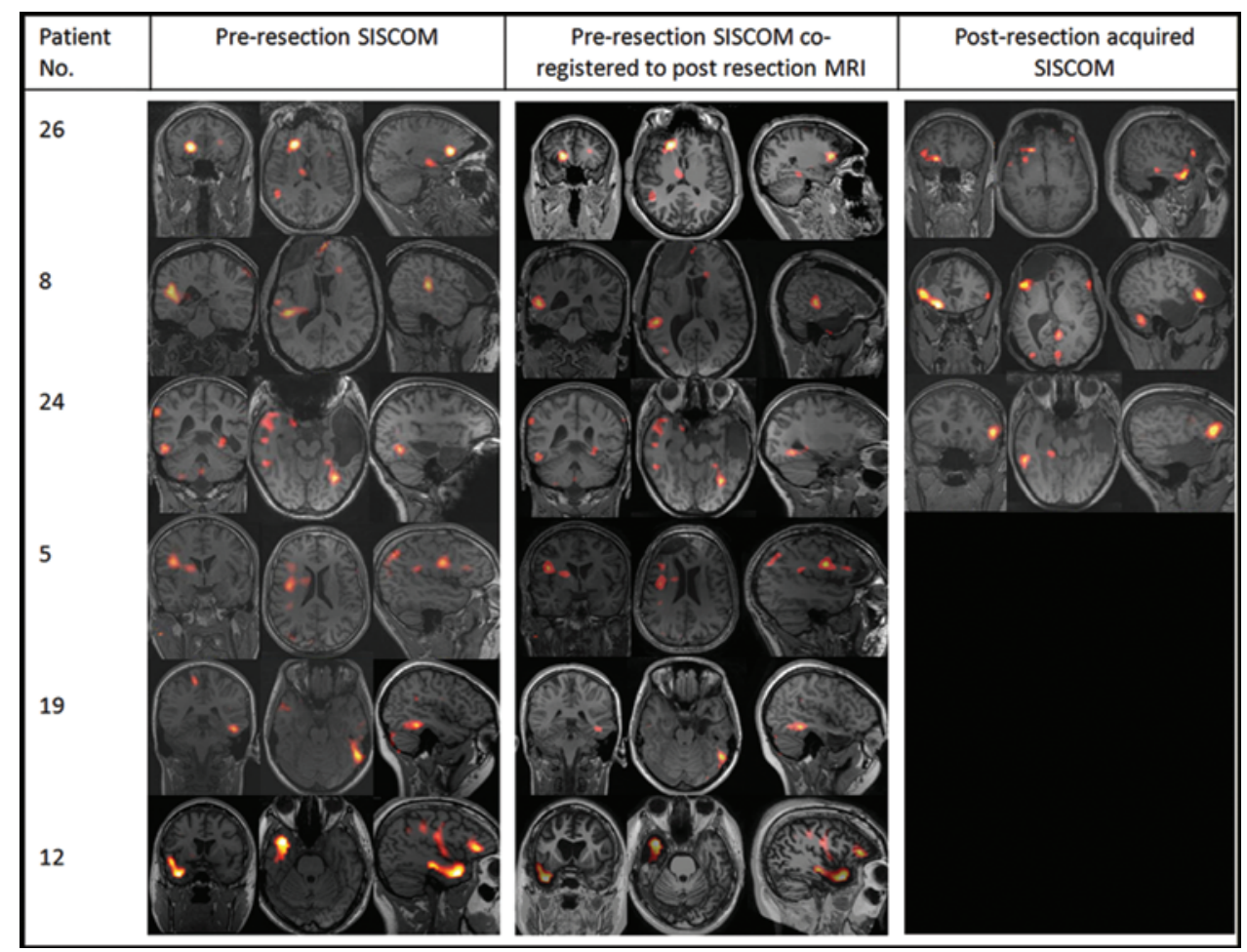

FIG. 2. Presentation of patients with SISCOM foci localizing with the resection site. These patients demonstrated poor surgical outcomes (Engel Class IV), and at least 1 SISCOM region did not overlap with the resection site. Figure is available in color online only. 
the utility of SISCOM by improving the outcomes of drugresistant, focal-onset epilepsy in both ETLE and TLE patients. Concordance between SISCOM and ECoG and/or $\mathrm{aECoG}$ in this refractory epilepsy cohort provides useful additional information for predicting postresection outcomes. In addition, the limitations in SPECT scanner technology may contribute to the sensitivity of SISCOM for identifying the maximal extent of the critical nodes or the generators of the epileptogenic circuit. The predominant limitation of conventional SPECT technology is the voxel resolution, which is limited to 8 to $12 \mathrm{~mm}$ with inadequate attenuation correction. The limitations of the spatial resolution of SPECT are related to using only 2- and 3-head photon detectors to record low-energy single-photon events. Next-generation SPECT scanner systems with significantly improved resolution and complementary postprocessing techniques will facilitate identifying a greater extent of the critical nodes in an epileptogenic network. ${ }^{16}$ Such technology will augment guiding ECoG electrode placement in potentially extensive epileptogenic networks.

\section{References}

1. Bell ML, Rao S, So EL, Trenerry M, Kazemi N, Stead SM, et al: Epilepsy surgery outcomes in temporal lobe epilepsy with a normal MRI. Epilepsia 50:2053-2060, 2009

2. Berg AT, Kelly MM: Defining intractability: comparisons among published definitions. Epilepsia 47:431-436, 2006

3. Berkovic SF, McIntosh AM, Kalnins RM, Jackson GD, Fabinyi GC, Brazenor GA, et al: Preoperative MRI predicts outcome of temporal lobectomy: an actuarial analysis. Neurology 45:1358-1363, 1995

4. Brodie MJ, Dichter MA: Antiepileptic drugs. N Engl J Med 334:168-175, 1996

5. Brodie MJ, Kwan P: Staged approach to epilepsy management. Neurology 58 (8 Suppl 5):S2-S8, 2002

6. Carne RP, O'Brien TJ, Kilpatrick CJ, MacGregor LR, Hicks RJ, Murphy MA, et al: MRI-negative PET-positive temporal lobe epilepsy: a distinct surgically remediable syndrome. Brain 127:2276-2285, 2004

7. Chung CK, Lee SK, Kim KJ: Surgical outcome of epilepsy caused by cortical dysplasia. Epilepsia 46 (Suppl 1):25-29, 2005

8. Cohen-Gadol AA, Wilhelmi BG, Collignon F, White JB, Britton JW, Cambier DM, et al: Long-term outcome of epilepsy surgery among 399 patients with nonlesional seizure foci including mesial temporal lobe sclerosis. J Neurosurg 104:513-524, 2006

9. Eliashiv SD, Dewar S, Wainwright I, Engel J Jr, Fried I: Long-term follow-up after temporal lobe resection for lesions associated with chronic seizures. Neurology 48:621-626, 1997

10. Elsharkawy AE, Alabbasi AH, Pannek H, Schulz R, Hoppe M, Pahs G, et al: Outcome of frontal lobe epilepsy surgery in adults. Epilepsy Res 81:97-106, 2008

11. Engel J Jr, Van NP, Rasmussen TB, Ojemann LM: Outcome with respect to epileptic seizures, in Engel J Jr (ed): Surgical Treatment of the Epilepsies. New York: Raven Press, 1993, pp 609-621

12. Ferrier CH, Alarcon G, Engelsman J, Binnie CD, Koutroumanidis M, Polkey CE, et al: Relevance of residual histologic and electrocorticographic abnormalities for surgical outcome in frontal lobe epilepsy. Epilepsia 42:363-371, 2001

13. Ferrier CH, Engelsman J, Alarcón G, Binnie CD, Polkey CE: Prognostic factors in presurgical assessment of frontal lobe epilepsy. J Neurol Neurosurg Psychiatry 66:350-356, 1999

14. Holmes MD, Born DE, Kutsy RL, Wilensky AJ, Ojemann
GA, Ojemann LM: Outcome after surgery in patients with refractory temporal lobe epilepsy and normal MRI. Seizure 9:407-411, 2000

15. Hong KS, Lee SK, Kim JY, Lee DS, Chung CK: Pre-surgical evaluation and surgical outcome of 41 patients with nonlesional neocortical epilepsy. Seizure 11:184-192, 2002

16. Jain M, Krug K, Balaguera P, Millan P, Jalota A, Pylypyuk $\mathrm{V}$, et al: Moving towards new techniques in the evaluation of the ictal onset zone: Ratio ictal SPECT (RISCOM) using a 72-detector focused collimator ring SPECT scanner system, Presented at the American Epilepsy Society 68th Annual Meeting, Seattle, 2014 (Abstract No. 2.241) (https:// www.aesnet.org/meetings_events/annual_meeting_abstracts/ view/1868323) [Accessed February 1, 2016]

17. Jeha LE, Najm I, Bingaman W, Dinner D, Widdess-Walsh P, Lüders H: Surgical outcome and prognostic factors of frontal lobe epilepsy surgery. Brain 130:574-584, 2007

18. Kutsy RL: Focal extratemporal epilepsy: clinical features, EEG patterns, and surgical approach. J Neurol Sci 166:1-15, 1999

19. Kwan P, Brodie MJ: Effectiveness of first antiepileptic drug. Epilepsia 42:1255-1260, 2001

20. Lee SK, Lee SY, Kim KK, Hong KS, Lee DS, Chung CK: Surgical outcome and prognostic factors of cryptogenic neocortical epilepsy. Ann Neurol 58:525-532, 2005

21. Mosewich RK, So EL, O'Brien TJ, Cascino GD, Sharbrough FW, Marsh WR, et al: Factors predictive of the outcome of frontal lobe epilepsy surgery. Epilepsia 41:843-849, 2000

22. Najm I, Jehi L, Palmini A, Gonzalez-Martinez J, Paglioli E, Bingaman W: Temporal patterns and mechanisms of epilepsy surgery failure. Epilepsia 54:772-782, 2013

23. Noe K, Sulc V, Wong-Kisiel L, Wirrell E, Van Gompel JJ, Wetjen N, et al: Long-term outcomes after nonlesional extratemporal lobe epilepsy surgery. JAMA Neurol 70:10031008, 2013

24. Radhakrishnan K, So EL, Silbert PL, Jack CR Jr, Cascino GD, Sharbrough FW, et al: Predictors of outcome of anterior temporal lobectomy for intractable epilepsy: a multivariate study. Neurology 51:465-471, 1998

25. Rasmussen T: Tailoring of cortical excisions for frontal lobe epilepsy. Can J Neurol Sci 18 (4 Suppl):606-610, 1991

26. Sander JW: Some aspects of prognosis in the epilepsies: a review. Epilepsia 34:1007-1016, 1993

27. Schmidt D, Gram L: Monotherapy versus polytherapy in epilepsy: a reappraisal. CNS Drugs 3:194-208, 1995

28. Simasathien T, Vadera S, Najm I, Gupta A, Bingaman W, Jehi L: Improved outcomes with earlier surgery for intractable frontal lobe epilepsy. Ann Neurol 73:646-654, 2013

29. Swartz BE, Delgado-Escueta AV, Walsh GO, Rich JR, Dwan PS, DeSalles AA, et al: Surgical outcomes in pure frontal lobe epilepsy and foci that mimic them. Epilepsy Res 29:97108, 1998

30. Sylaja PN, Radhakrishnan K, Kesavadas C, Sarma PS: Seizure outcome after anterior temporal lobectomy and its predictors in patients with apparent temporal lobe epilepsy and normal MRI. Epilepsia 45:803-808, 2004

31. Téllez-Zenteno JF, Hernández Ronquillo L, Moien-Afshari F, Wiebe S: Surgical outcomes in lesional and non-lesional epilepsy: a systematic review and meta-analysis. Epilepsy Res 89:310-318, 2010

32. Theodore WH, Spencer SS, Wiebe S, Langfitt JT, Ali A, Shafer PO, et al: Epilepsy in North America: a report prepared under the auspices of the global campaign against epilepsy, the International Bureau for Epilepsy, the International League Against Epilepsy, and the World Health Organization. Epilepsia 47:1700-1722, 2006

33. Wetjen NM, Cascino GD, Fessler AJ, So EL, Buchhalter JR, Mullan BP, et al: Subtraction ictal single-photon emission computed tomography coregistered to magnetic resonance 
imaging in evaluating the need for repeated epilepsy surgery. J Neurosurg 105:71-76, 2006

34. Wieshmann UC, Larkin D, Varma T, Eldridge P: Predictors of outcome after temporal lobectomy for refractory temporal lobe epilepsy. Acta Neurol Scand 118:306-312, 2008

35. Yun CH, Lee SK, Lee SY, Kim KK, Jeong SW, Chung CK: Prognostic factors in neocortical epilepsy surgery: multivariate analysis. Epilepsia 47:574-579, 2006

36. Zaatreh MM, Spencer DD, Thompson JL, Blumenfeld H, Novotny EJ, Mattson RH, et al: Frontal lobe tumoral epilepsy: clinical, neurophysiologic features and predictors of surgical outcome. Epilepsia 43:727-733, 2002

\section{Disclosures}

Dr. Byrne reports that he is a consultant for Stryker and Integra.

\section{Author Contributions}

Conception and design: Jalota, Rossi, Byrne. Acquisition of data: Stein, Stoub, Balabanov, Bergen, Bermeo, Park, Smith. Analysis and interpretation of data: Jalota, Rossi. Drafting the article: Jalota, Rossi. Critically revising the article: Jalota, Rossi. Reviewed submitted version of manuscript: Jalota, Rossi. Byrne. Approved the final version of the manuscript on behalf of all authors: Rossi. Statistical analysis: Jalota, Rossi, Pylypyuk. Administrative/technical/material support: Rossi, Pylypyuk. Study supervision: Rossi.

\section{Correspondence}

Marvin A. Rossi, Department of Neurological Sciences, Rush University Medical Center, 1725 W. Harrison St., Ste. 885, Chicago, IL 60612.email: marossi@usa.net. 